\title{
Overview of trochanteric fractures treated surgically
}

\author{
Thong Nguyen ${ }^{1}$, Quan Nguyen ${ }^{1}$, and Dung Nguyen ${ }^{1}$ \\ ${ }^{1}$ Affiliation not available
}

May 12, 2020

\begin{abstract}
The trochanteric fractures usually occur in elderly people who are often associated with osteoporosis; the impacting forces are commonly low energy. The displacement of fragments can be much or little, but it can cause pain a lot.
\end{abstract}

\section{Overview of trochanteric fractures treated surgically}

Nguyen Vinh Thong | Nguyen Minh Quan | Nguyen Xuan Dung

Thu Duc District Hospital, Ho Chi Minh city, Vietnam.

Correspondence

Nguyen Vinh Thong, Orthopedic department, Thu Duc District Hospital, Ho Chi Minh city, Vietnam.

Email: nguyenvinhthong51@gmail.com

\section{Key Clinical Message}

The surgical way is usually chosen differently from personal experience of surgeon. The author has presented the guidelines for choosing surgical method from three well-known organizations: AAOS of US, Sofcot of France, and International AO. The choosing is based on personal fracture lines, patient status, functional level of hip joint.

\section{1 | INTRODUCTION}

Trochanteric fractures are extra-capsular fractures of the proximal femur between the greater and lesser trochanters, usually occur in the elderly, women are more than men, often more than 2 times.

The mechanism of injury for young people is often due to high energy, while for older people due to low energy. Trochanteric fractures if treated early, the risk of non-union or mal-union is low.

The surgical management of fractures needs to coordinate treatment of accompanying medical conditions, otherwise the death rate in the first year is $20-30 \%$ [1].

\section{2 | TROCHANTERIC FRACTURE CLASSIFICATION}

There are many different classifications of the trochanteric fractures, where we use the AO or Muller classification, which most studies have used.

Type A1: Pertrochanteric fractures are simple with 2 fragments. (Figure 1)

- Type A1.1: Pertrochanteric fractures, small displaced.

- Type A1.2: Pertrochanteric fractures, with wide displacement at greater trochanter.

- Type A1.2: The fracture line has spread to lesser trochanter.

Fracture type A2: Pertrochanteric fractures are multi-fragmentary. (Figure 1) 
- Type A2.1: Detachment of the lesser trochanter.

- Type A2.2: Several intermediate fragments including detachment of the lesser trochanter.

- Type A2.3: Several intermediate fragments extending more than $1 \mathrm{~cm}$ distal to the lesser trochanter.

Type A3: Intertrochanteric fractures all have a fracture line through the lateral femoral wall. (Figue 3)

- Type A3.1: simple fracture, reverse oblique fracture line.

- Type A3.2: Simple fracture, transverse line.

- Type A3.3: Simple transverve line with medial detached fragment.

\section{3 | STABLE AND UNSTABLE TROCHANTERIC FRACTURES}

The trochanteric fractures are considered stable if after reduction and fixation are less likely to be secondary displaced [1].

- Stable fracture: with intact posteromedial cortex that will resist medial compressive loads once reduced.

- Unstable fracture: comminuted fracture of the posteromedial cortex, thinner lateral wall thickness, the fracture will collapse into varus and retroversion when loaded. For instance fractures with a large posteromedial fragment; reverse oblique fracture line extending from medial cortex both laterally and distally; sub-trochanteric extension.

\section{4 | NONOPERATIVE TREATMENT}

Indications [1]:

- Non-ambulatory patients.

- Patients at high risk for perioperative mortality. Allow the patient to sit and stand early but not weight bearing.

\section{Outcomes:}

- High rates of pneumonia.

- Urinary tract infections.

- Decubitus ulcers, and DVT.

\section{5 | OPERATIVE TREATMENT}

Mainly apply 4 methods: Sliding hip compression screw (DHS), Intra-medullary hip screw (cephalomedullary nail), Hip replacement and Interlocking plates. Particularly, the interlocking plates for the proximal femoral head we have not discussed because so far the results of postoperative monitoring in many centers have not been good [1].

\section{Sliding hip compression screw (DHS): (Figue 4)}

Indicated for cases of fracture type A1 and A2. For stable fractures, if reduction and fixation in good quality the fractures will be healed as well as Intramedullary hip screw.

\section{Technique:}

- Must obtain correct neck-shaft relationship.

- Lag screw with tip-apex distance $>25 \mathrm{~mm}$ is associated with increased failure rate.

- 4 hole plates show no benefit clinically or biomechanically over 2 hole plates.

Pros:

- Allows dynamic inter-fragmentary compression.

- Low cost.

- No violation of hip abductors.

Cons: 
- Being an open surgical technique, the possibility of blood loss.

- Not being advisable in unstable fracture patterns because of risks of collapse of fracture area; limb shortening and medial shifting of the femoral shaft.

Complications:

The sliding screw "cut out" the head bone, usually occurs within first 3 months. If tip-apex distance $>45$ $\mathrm{mm}$ associated with $60 \%$ failure rate.

Intramedullary hip screw (Cephalomedullary nail): (Figue 5)

\section{Technique:}

- Short intramedullary nail with distal locking for standard obliquity fractures.

- Long intramedullary nail for reverse obliquity fracture and sub-trochanteric extension.

Pros:

- Percutaneous approach.

- Minimal blood loss.

- May be used in unstable fracture patterns.

Cons:

- Peri-prosthetic fracture.

- Higher cost than sliding hip screw.

- Requires violation of hip abductors for insertion.

Complications:

Anterior perforation of the distal femur can occur following intramedullary screw fixation, the causes may be due to mismatch of the radius of curvature of the femur and implant or posterior starting point on the greater trochanter.

Hip replacement: (Figure 6)

Indications

- Severely comminuted fractures.

- Preexisting symptomatic degenerative arthritis.

- Osteoporotic bone that is unlikely to hold internal fixation.

- Salvage for failed internal fixation.

Technique :

- Reduction of calcar fragment or replacing prosthesis.

- Must attempt fixation of greater trochanter to shaft.

Pros: possible earlier return for full weight bearing.

Cons:

- Blood loss may occur.

- Demanding surgical technique in comparison to usual.

5 | RECOMMENTATION OF THE AMERICAN ACADEMY OF ORTHOPAEDIC SURGEONS (AAOS) 2015

The Board recommended the surgical management of the fractures in different scenarios, we only cite 4 situations related to trochanteric fractures in the elderly, the stability of the fracture, the status of the hip is in good condition or has degeneration, walking requires more or less [2]. (Table $1-4$ ) 


\section{7 | RECOMMENTATION OF THE ORTHOPAEDIC ASSOCIATION OF FRANCE (SOF- COT) 2016}

The purpose of this study was to determine whether there is room for hip replacement surgery in the treatment of extra-capsular proximal femur fractures in elderly patients [3].

To answer this question, the following criteria were analyzed for both techniques:

- Functional results

- Duration of operation - length of hospital stay

- Blood loss

- Mechanical complication, morbidity and mortality

All these data do not make it possible to determine whether the treatment of per-trochanteric fractures by hip replacement would have an advantage over bone fixation treatment.

Nevertheless, as in the international recommendations, bone fixation remains recommended in the treatment of extra-capsular fractures of the proximal end of the femur. In cases where it cannot be considered, hip replacement will be performed.

The reasons for the indication of hip replacement (comorbidity, state of the joint, etc.) should be mentioned in the patient's medical file.

\section{8 | RECOMMENTATION OF INTERNATIONAL AO FOUNDATION}

Trochanteric fracture divides into 3 types [4]:

- Pertrochanteric, simple.

- Pertrochanteric, multi-fragmentary.

- Intertrochanteric.

Pertrochanteric, simple fractures : (Figue 7)

- These fractures cause significant shortening, but they are stable after reduction and fixation, largely because of the excellent contact of the fracture surfaces

- The lesser trochanter, the so-called medial buttress, is intact.

- These fractures may be treated with a sliding hip screw and plate, or a cephalomedullary nail.

Pertrochanteric, multi-fragmentary fractures: (Figue 8)

- In multi-fragmentary per-trochanteric fractures the fracture line can start laterally anywhere on the greater trochanter and runs towards the medial cortex which is broken in two places. This results in the detachment of a third fragment which includes the lesser trochanter.

- These fractures may be treated with a sliding hip screw and plate, or a cephalomedullary nail

Intertrochanteric fractures: (Figue 9)

- True intertrochanteric fractures are subdivided according to the fracture pattern. The fracture line passes between the two trochanters, above the lesser trochanter medially and below the crest of the vastus lateralis laterally. Both femoral cortices are involved.

- These fractures may be treated with a sliding hip screw and plate (DCS), or a cephalomedullary nail.

\section{9 | SOME COMMENTS ABOUT SURGICAL TREATMENT}

Most femoral trochanteric fractures are treated surgically, if there is no contraindication due to the patient's health status.

In terms of surgical methods, until now, the bone fixation is still considered the mainstream, in which mainly the sliding hip compression screw (DHS) and the intramedullary hip screw nail, while the hip replacement 
is indicated in special cases are related to the patient's overall condition as well as the functional status of the hip.

- Sliding hip screw is indicated when the fracture line is stable, according to AO classification as type A1 and A2.1, A2.2, and when the lateral wall of the femoral shaft is intact and has thickness bigger than $2 \mathrm{~cm}$.

- Intramedullary hip screw is widely indicated, including both stable and unstable fractures

- Hip replacement is indicated when the hip joint has been pre-existing and symptomatic arthritis, or when the fracture is severe comminuted not able to do bone fixation.

\section{0 | A PILOT STUDY AT SAIGON ITO HOSPITAL}

Data collected from 2018 and the first half of 2019: 80 cases

Male: 25 case; Female: 55 case. (Table 5)

Surgical method:

- Interlocking plate: 5 cases.

- Sliding hip compression screw: 22 case.

- Intramedullary hip screw: 22 case.

- Hip replacement: 31 case. Hip replacement is indicated when the hip joint has been pre-existing and symptomatic arthritis, or when the fracture line is severe comminuted not able to do bone fixation.

From the data we found that:

- Frequency of using the DHS and intramedullary hip screw is equal (27.5\%).

- Hip replacement is a little more with $38.7 \%$.

- Interlocking plate is used the least $(6.3 \%)$.

- Most people younger than 60 of age operated with DHS or intramedullary hip screw

- People more than 80 years old, almost treated with hip replacement or intramedullary hip screw. Varied methods used for dealing with trochanteric fractures at Saigon-Ito hospital seem to be diversity. Although the number of hip replacement is a little more than that of bone fixation, the data also reflect the application of treatment facilities quite similar to the general trend of developed countries.

\section{1 | CONCLUSION}

Almost trochanteric displaced fractures need to be operated.

There are 4 current methods of surgery sorted in descending order of priority: 1. Intramedullary hip screw 2. Sliding hip compression screw (DHS) 3. Hip replacement 4. Interlocking plate.

Selecting a surgical method should base on:

- Structure of fracture lines.

- Degenerative status of hip joint.

- Daily activities, including walking.

- Health status of patient.

\section{Author Contribution}

Nguyen Vinh Thong and Nguyen Minh Quan conceived of the presented idea. Nguyen Vinh Thong developed the theory and performed the computations. Nguyen Xuan Dung verified the analytical methods. Nguyen Minh Quan encouraged Nguyen Vinh Thong to investigate and supervised the findings of this work. All authors discussed the results and contributed to the final manuscript.

\section{References}

1. Karadsheh MS. Intertrochanteric fractures Ortho Bullets 2019 [updated 5/01/20120; cited 10/5/2020. Available from: https://www.orthobullets.com/trauma/1038/intertrochanteric-fractures. 
2. American Academy of Orthopaedic Surgeons. Appropriate use criteria for the treatment of hip fractures in the elderly. Adopted by the American Academy of Orthopaedic Surgeons Board of Directors2015.

3. Haute Autorité de Santé. Chirurgie des fractures de l'extrémité proximale du fémur chez lespatients âgés - Méthode Recommandations pour la pratique clinique. 2016.

4. Mattos C, Jesus A, Floter M, Nunes L, Sanches B, Zabeu J. Reproducibility of the Tronzo and AO classifications for transtrochanteric fractures. Revista Brasileira de Ortopedia (English Edition). 2015;50.

5. Raaymakers E, Schipper I, Simmermacher R, Werken Cvd. Trochanteric fracture, pertrochanteric, simple 2010 [Available from: https://surgeryreference.aofoundation.org/orthopedic-trauma/adulttrauma/proximal-femur/trochanteric-fracture-pertrochanteric-simple.

6. Raaymakers E, Schipper I, Simmermacher R, Werken Cvd. Trochanteric fracture, pertrochanteric, multifragmentary 2010 [Available from: https://surgeryreference.aofoundation.org/orthopedic-trauma/adult-trauma/proximal-femur/trochantericfracture-pertrochanteric-multifragmentary?searchurl $=\% 2 \mathrm{fSearchResults.}$

7. Raaymakers E, Schipper I, Simmermacher R, Werken Cvd. Trochanteric fracture, intertrochanteric 2010 [Available from: https://surgeryreference.aofoundation.org/orthopedic-trauma/adulttrauma/proximal-femur/trochanteric-fracture-intertrochanteric?searchurl=\%2fSearchResults.

\section{LIST OF FIGURE AND TABLE LEGENDS}
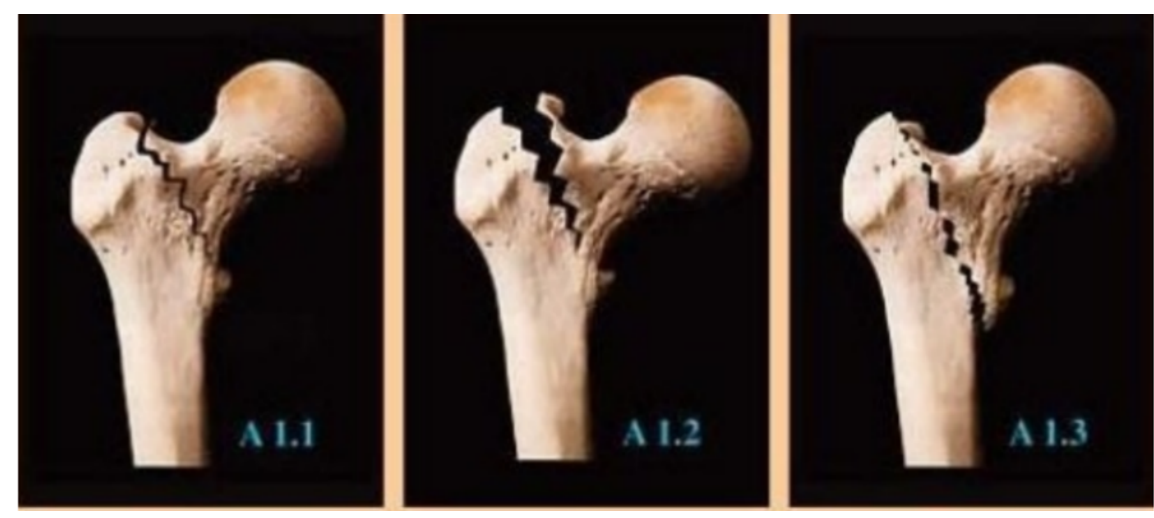

FIGURE 1: Fracture type A1: A1.1, A1.2 and A1.3 [4]
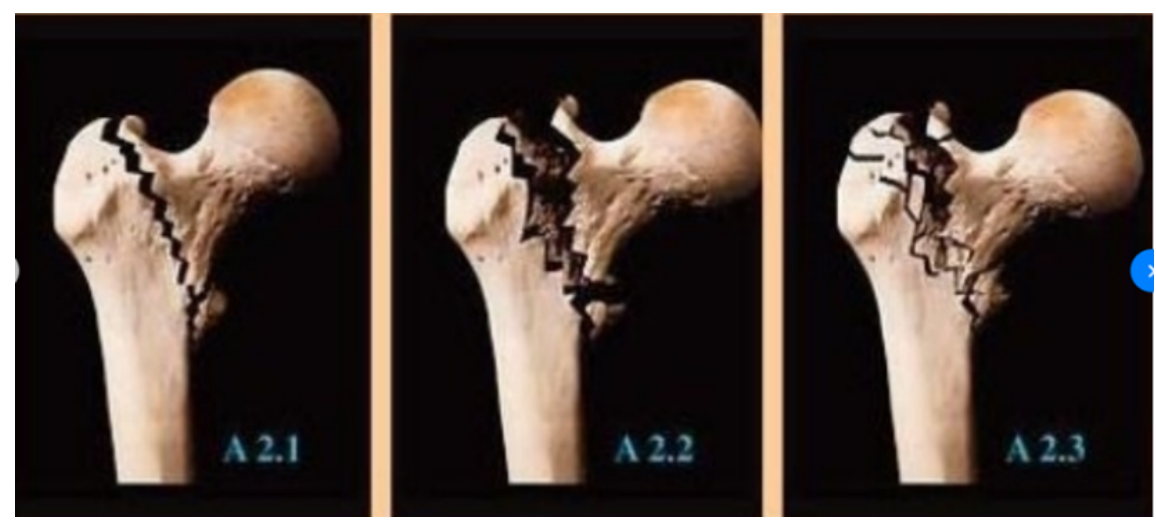

FIGURE 2 : Fracture type A2: A2.1, A2.2 and A2.3 [4] 

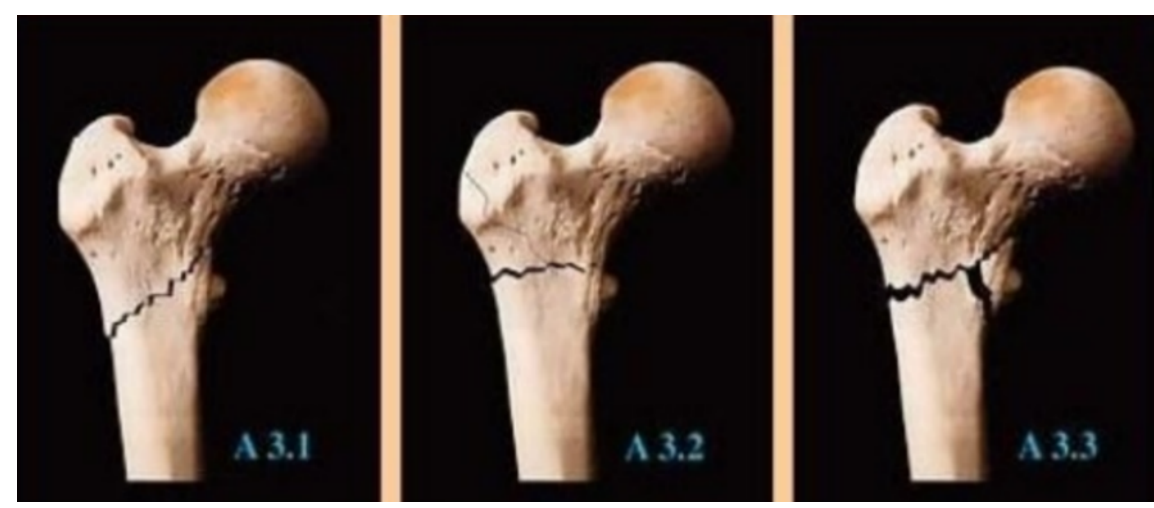

FIGURE 3 : Fracture type A3: A3.1, A3.2 and A3.3 [4]

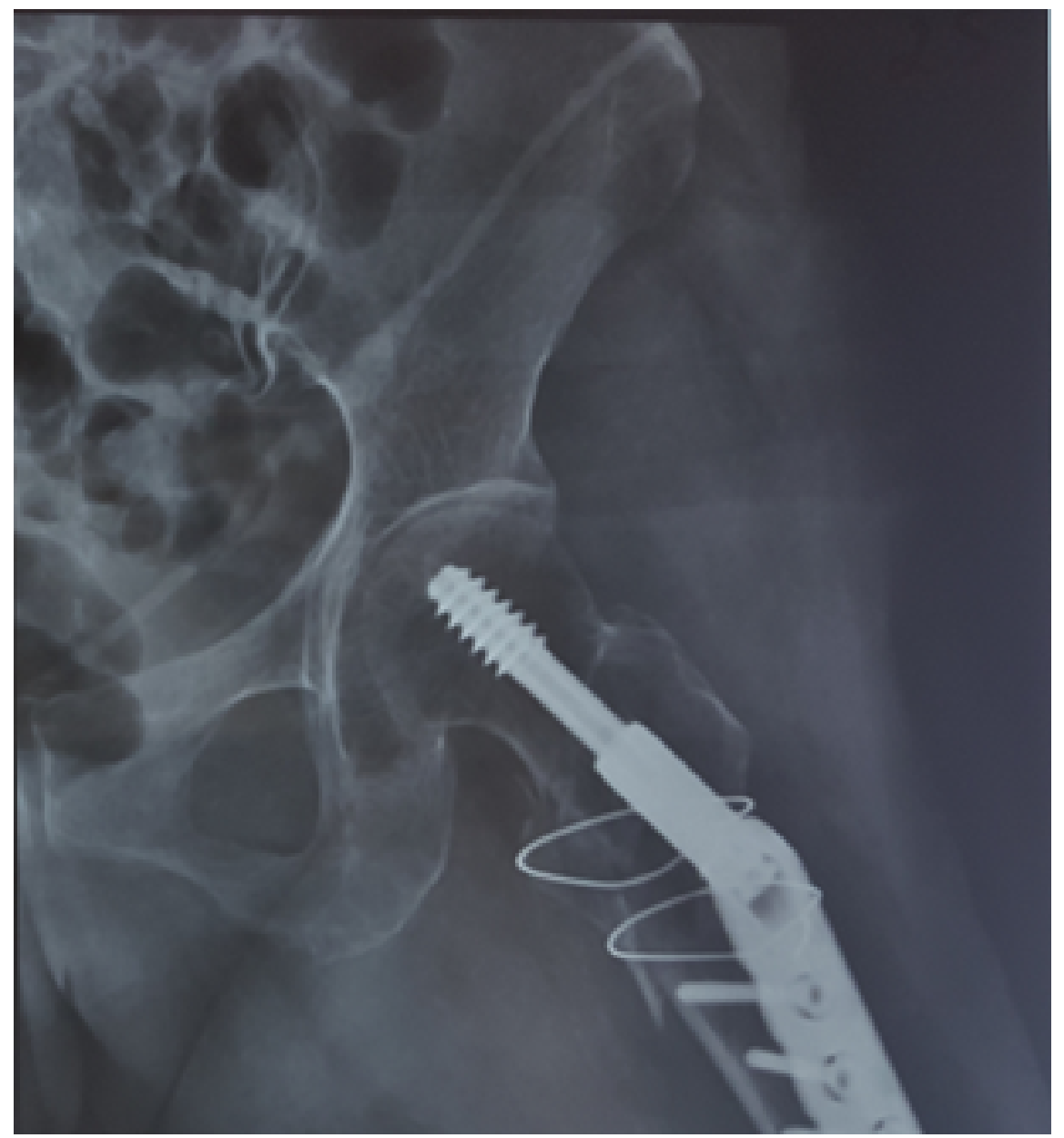

FIGURE 4 : Patient 65 years old, fracture type A2.2, operated with DHS 


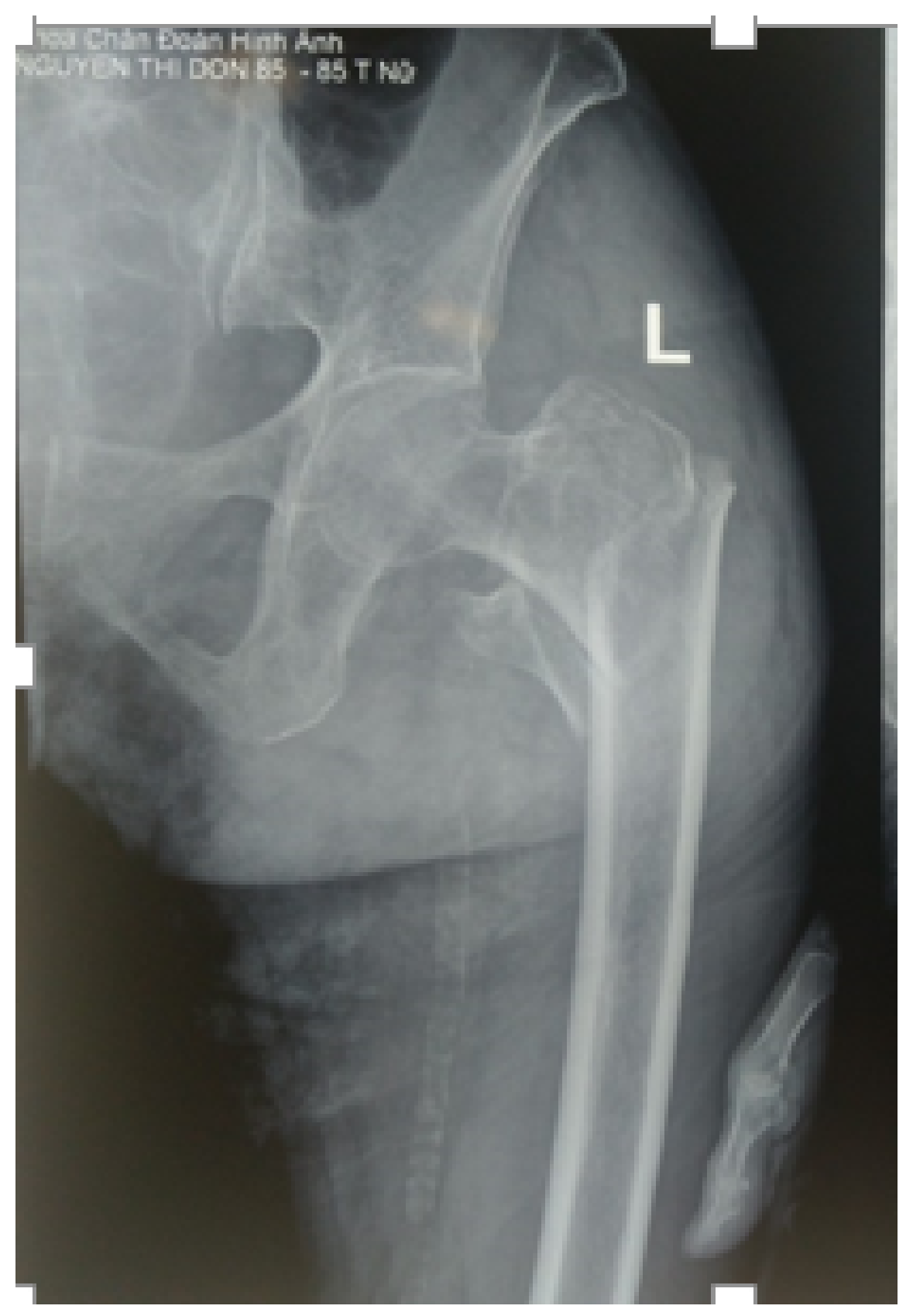

FIGURE 5: Patient 85 years old, fracture type A2.3, operated with intramedullary hip screw 


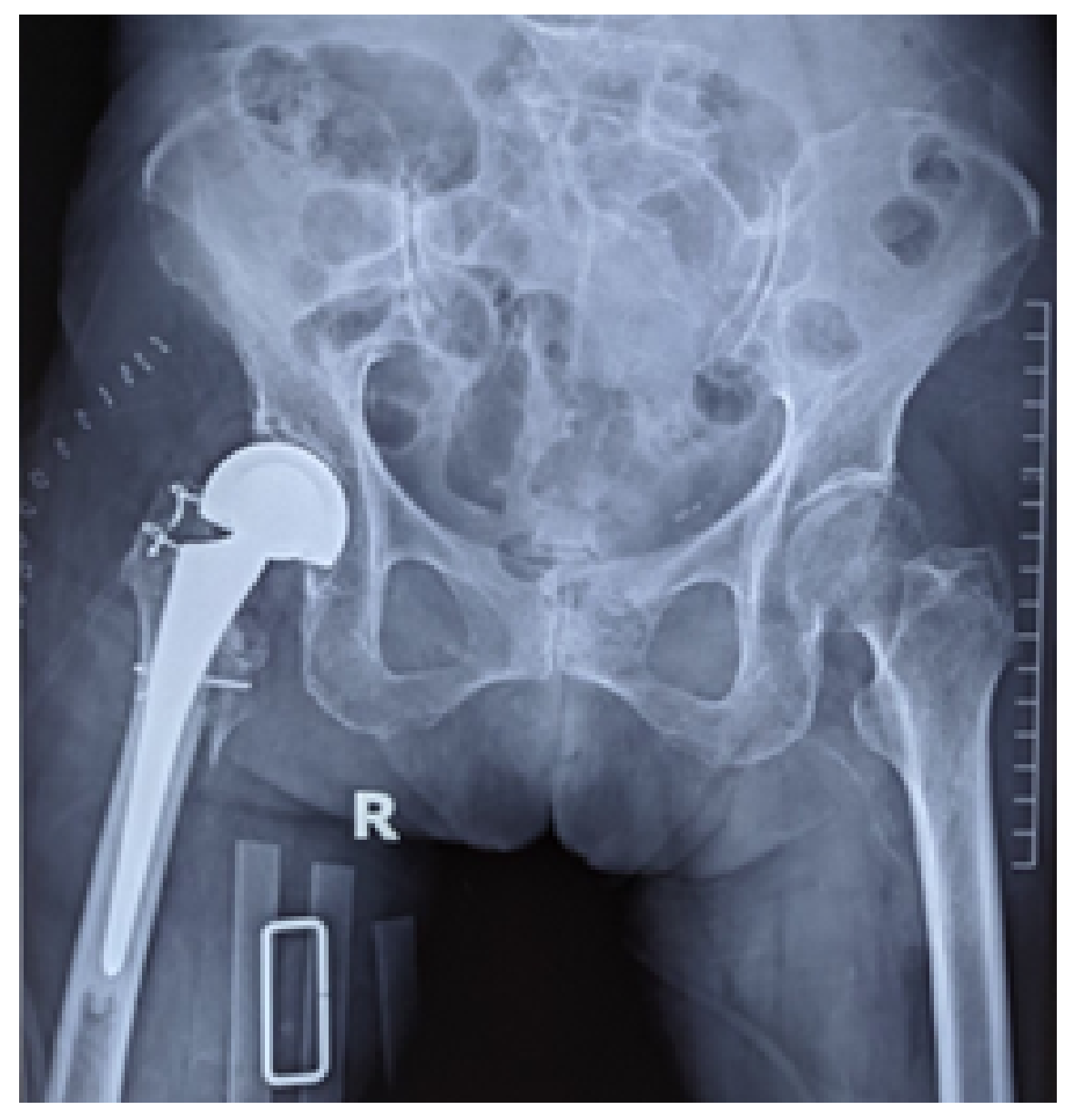

FIGURE 6: Patient 95 years old, fracture type A2.3, operated with cemented bipolar hip replacement 


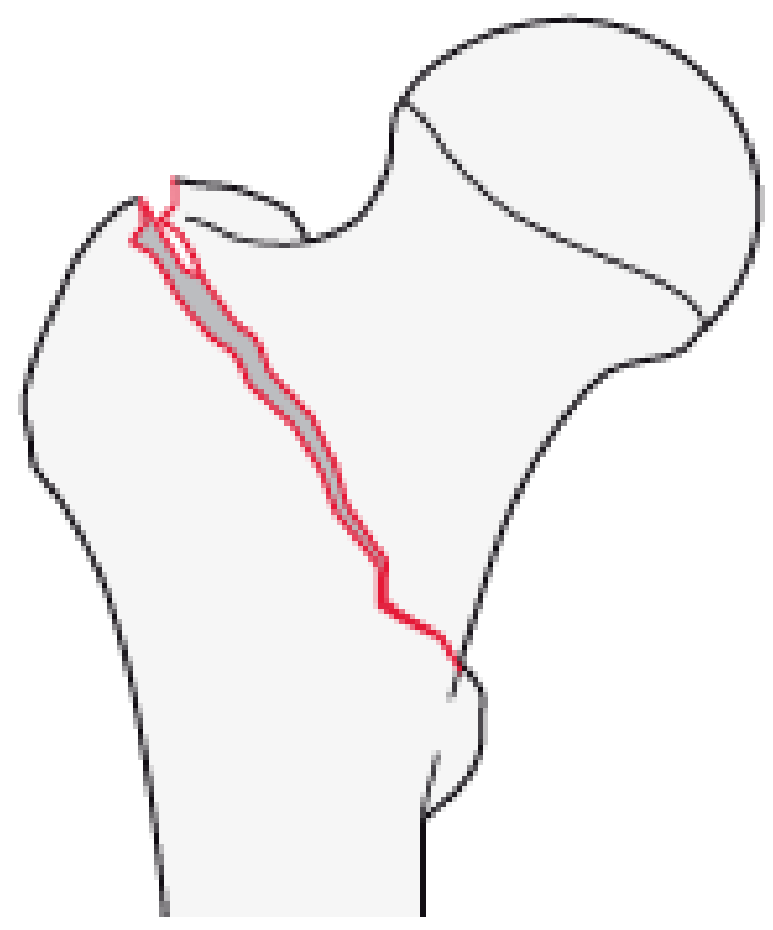

FIGURE 7: Pertrochanteric, simple fracture [5]

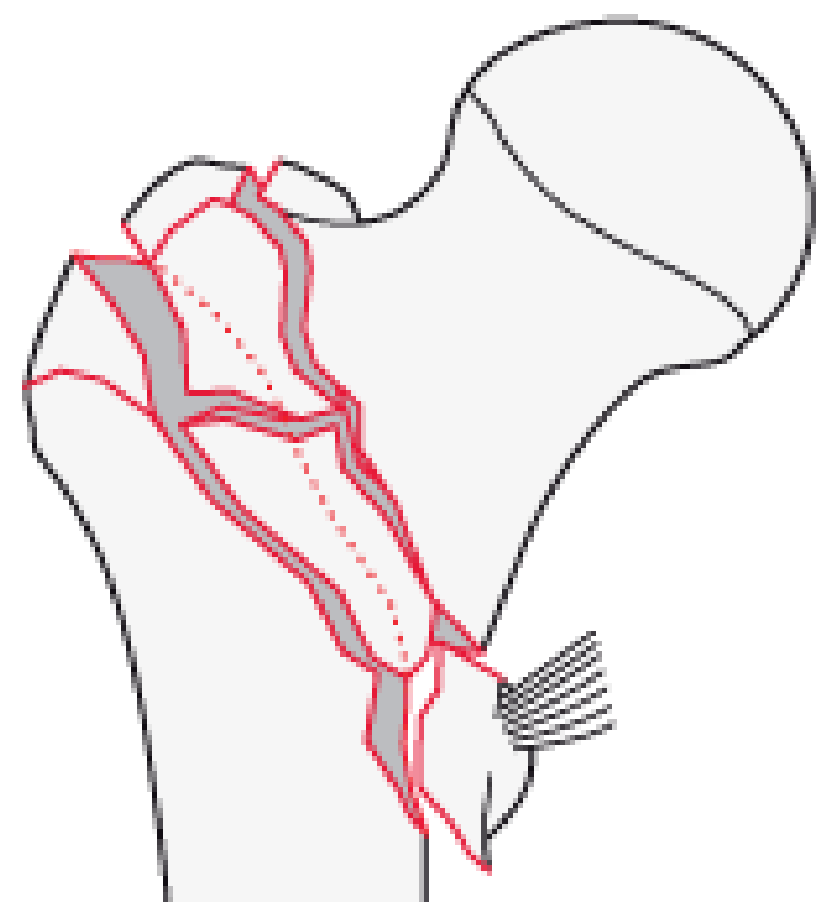

FIGURE 8: Pertrochanteric, multifragmentary fracture [6] 


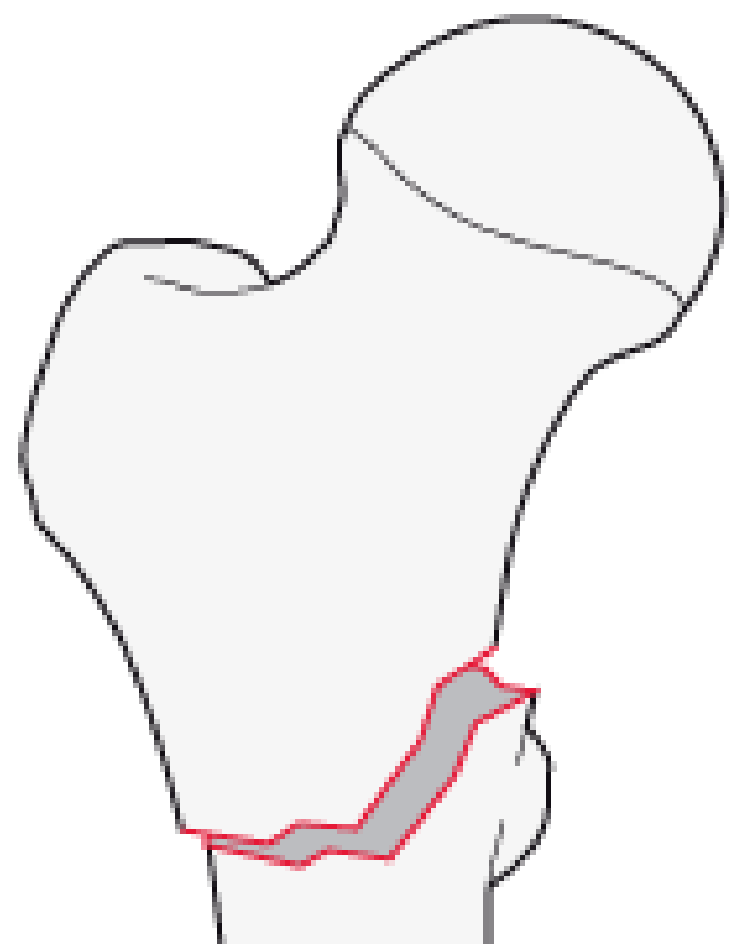

FIGURE 9: Intertrochanteric fracture [7]

Table 1: Stable Intertrochanteric, High functioning/high demand patient, Preexisting and Symptomatic Arthritis

\begin{tabular}{lllll}
\hline Treatment & Appropriateness & M & A & Agreement \\
\hline Total Hip Arthroplasty & May Be Appropriate & 4 & $\mathrm{~N}$ & \\
Hemiarthroplasty & Rarely Appropriate & 2 & $\mathrm{~A}$ & + \\
Long Cephalomedullary Nails & May Be Appropriate & 6 & $\mathrm{~N}$ & \\
Short Cephalomedullary Nails & Appropriate & 7 & $\mathrm{~N}$ & \\
Sliding Hop Screw \pm Anti-Rotation Screw & Appropriate & 9 & $\mathrm{~A}$ & + \\
Multiple Screw Fixation & Rarely Appropriate & 1 & $\mathrm{~A}$ & + \\
\hline
\end{tabular}

Table 2: Stable Intertrochanteric, High functioning/high demand patient, No Preexisting and Symptomatic Arthritis

\begin{tabular}{lllll}
\hline Treatment & Appropriateness & M & A & Agreement \\
\hline Total Hip Arthroplasty & May Be Appropriate & 1 & $\mathrm{~N}$ & + \\
Hemiarthroplasty & Rarely Appropriate & 1 & $\mathrm{~A}$ & + \\
Long Cephalomedullary Nails & May Be Appropriate & 4 & $\mathrm{~N}$ & \\
Short Cephalomedullary Nails & Appropriate & 7 & $\mathrm{~N}$ & \\
Sliding Hop Screw \pm Anti-Rotation Screw & Appropriate & 9 & $\mathrm{~A}$ & + \\
Multiple Screw Fixation & Rarely Appropriate & 1 & $\mathrm{~A}$ & + \\
\hline
\end{tabular}

Table 3: Unstable Intertrochanteric, High functioning/high demand patient, Preexisting and Symptomatic 
Arthritis

\begin{tabular}{lllll}
\hline Treatment & Appropriateness & M & A & Agreement \\
\hline Total Hip Arthroplasty & May Be Appropriate & 3 & $\mathrm{~N}$ & + \\
Hemiarthroplasty & Rarely Appropriate & 3 & $\mathrm{~A}$ & \\
Long Cephalomedullary Nails & May Be Appropriate & 8 & $\mathrm{~N}$ & + \\
Short Cephalomedullary Nails & Appropriate & 7 & $\mathrm{~N}$ & \\
Sliding Hop Screw \pm Anti-Rotation Screw & Appropriate & 5 & $\mathrm{~A}$ & \\
Multiple Screw Fixation & Rarely Appropriate & 1 & $\mathrm{~A}$ & + \\
\hline
\end{tabular}

Table 4: Unstable Intertrochanteric, Moderate to low functioning patient, Preexisting and Symptomatic Arthritis

\begin{tabular}{lllll}
\hline Treatment & Appropriateness & M & A & Agreement \\
\hline Total Hip Arthroplasty & May Be Appropriate & 5 & $\mathrm{~N}$ & \\
Hemiarthroplasty & Rarely Appropriate & 4 & $\mathrm{~A}$ & \\
Long Cephalomedullary Nails & May Be Appropriate & 8 & $\mathrm{~N}$ & + \\
Short Cephalomedullary Nails & Appropriate & 7 & $\mathrm{~N}$ & + \\
Sliding Hop Screw \pm Anti-Rotation Screw & Appropriate & 6 & $\mathrm{~A}$ & \\
Multiple Screw Fixation & Rarely Appropriate & 1 & $\mathrm{~A}$ & + \\
\hline
\end{tabular}

Table 5: Age groups

\begin{tabular}{ll}
\hline Age groups & $\mathrm{N}$ \\
\hline $40-50$ & 4 \\
$50-60$ & 6 \\
$60-70$ & 12 \\
$70-80$ & 15 \\
$80-90$ & 32 \\
$90-100$ & 8 \\
$>100$ & 3 \\
\hline
\end{tabular}

